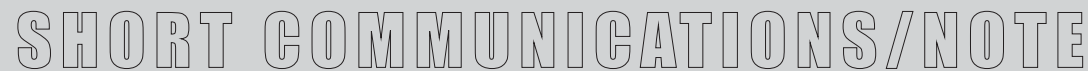

\section{Parassitosi intestinali umane nel Perugino nel corso del 2005}

\author{
Maria Letizia D'Annibale', Daniele Crotti²
}

'Struttura Complessa di Microbiologia, Azienda Ospedaliera di Perugia

${ }^{2}$ L. P. \& L. D. in Parassitologia e Microbiologia Medica, Perugia

Key words: helminths, intestinal parasitosis, O\&P, protozoa

Human intestinal parasitosis in Perugia during 2005

\section{SUMMARY}

During 2005 we analyzed stool specimens of 66 I subjects, 22 I children ( I - I6 years old) and 440 adults, for O\&P (direct and after formalin-etylacetate concentration microscopic observations, Giemsa and modified ZiehlNeelsen permanent stains when requested). $13.8 \%$ of subjects were positive for parasitic infections (I3.5\% among children and $13.9 \%$ among adults). $8.8 \%$ of subjects are infected with pathogen parasites (7.2\% among children and $9.6 \%$ among adults) and $5.0 \%$ with not pathogens (6.3\% and $4.3 \%$ respectively). Among pathogen parasites, in children G. duodenalis was observed in $4.1 \%$ of cases, D. fragilis in $0.5 \%$, E. vermicularis in $0.9 \%$, T. trichiura in $1.8 \%$. Among adults, D. fragilis was observed in $5.2 \%$ of cases, G. duodenalis in $1.8 \%$, Cryptosporidium spp. in $0.5 \%$, E. vermicularis in $0.5 \%$, T. trichiura in $0.7 \%$, S. stercoralis in $0.7 \%, H$. nana in $0.2 \%$, T. saginata in $0.5 \%$, S. mansoni in $0.5 \%$. Among childrens, $76.7 \%$ of pathogen parasites were imported from developing countries, particularly $G$. duodenalis in adopted ones; instead, among adults, $83.6 \%$ of pathogens was observed in home/ltalian people, particularly D. fragilis. Cellophane tape test was performed on 40 home children and E. vermicularis prevailed in $22 \%$; modified Baermann method was performed on 42 old subjects and S. stercoralis rabdithoid larvae were observed in $7.1 \%$ (but the same ones of O\&P). The Authors recommend the O\&P in subjects with intestinal aspecific troubles, ipereosinophilia, or other justified situations, emphasizing the importance of a rational, good and responsible O\&P and/or other techniques for intestinal parasitosis, because are present, even if not frequent, not only imported, particularly D. fragilis, but also helminths, and not only E. vermicularis.

\section{INTRODUZIONE}

Le parassitosi intestinali sono in Italia tutt'ora relativamente infrequenti rispetto a quei Paesi (spesso definiti in via di sviluppo tecnologico, PVS) in cui svariati fattori, soprattutto la povertà da cui le soventi precarie condizioni igienicosanitarie, ne condizionano in modo assai meno marcato la presenza $(7,8,28)$.

I dati reperibili in letteratura relativi alle parassitosi nell'uomo, nel nostro Paese, non sono numerosi; e questo proprio in considerazione, forse, della loro rarità (reale o presunta) o, comunque, della scarsa attenzione rivolta a tale problematica $(1,23)$.

Ciononostante, laddove la diagnostica di laboratorio di tali parassitosi viene eseguita routinariamente e con criteri metodologici soddisfacenti, le prevalenze delle medesime non sembrano essere così basse $(12,13)$. Vero è che più spesso ciò è legato al fatto che una quota non irrilevante di tali enteroparassitosi sono appannaggio di popolazioni o di campioni di popolazione provenienti da Paesi a maggiore endemia (PVS) e quindi importate (emigranti ed immigrati, nomadi, bambini adottati, viaggiatori), però è altrettanto vero che ciò è o può essere in relazione alla popolazione squisitamente autoctona, e questo sia in conseguenza della presenza o dell'aggravarsi di fattori di rischio (in primis l'immunodepressione) sia in virtù di maggiori e migliori attenzioni ai problemi anche diagnostici relativi a tali patologie, di indubbio interesse anche in ambito di sanità pubblica $(6,9,25)$. Ma, se l'approccio metodologico a siffatta diagnostica è razionale, cosciente e responsabile, e quindi anche le scelte tecniche ne risultano mirate e diversificate (conseguentemente più approfondite ed ampie), le frequenze di reperimento di parassiti a livello intestinale risultano più elevate $o$ comunque maggiormente diversificate $(14,19)$.

In tale presentazione vogliamo riportare $\mathrm{i}$ dati riguardanti un anno di sorveglianza diagnostica delle parassitosi intestinali nella nostra area geografica, e fornire al lettore e ai colleghi spunti di riflessione sia in merito ai percorsi diagnostici in senso stretto intesi sia in merito alle correlazioni clinicoepidemiologiche osservate al riguardo $(6,14,19)$.

Abbiamo pensato di presentare i risultati nel loro complesso, inizialmente, differenziando tra popolazione pediatrica e soggetti adulti, e quindi analizzare nel dettaglio le variabilità interne, spesso correlabili alla provenienza dei soggetti parassita- 
ti, ma altresì agli aspetti clinici e alle scelte diagnostiche operate $(2,3,11)$.

Tale indagine è stata di tipo retrospettivo, e si è basata sull'analisi dei risultati dei singoli campioni analizzati per ciascun soggetto, rilevando tutte le informazioni utili se non necessarie (anamnestiche, cliniche, epidemiologiche) dalle relative schede cliniche che accompagnavano i singoli campioni fecali $(14,19)$. Pertanto sono state escluse da tali valutazioni tutte quelle schede (e quindi campioni) relative a soggetti per i quali o mancavano le dovute ed indispensabili indicazioni in merito o si riferivano a controlli dopo terapie o in pazienti con terapia in atto o comunque erano ritenute non idonee per un'adeguata e congrua valutazione specifica (6).

\section{MATERIALI E METODI}

Nel corso del 2005 sono stati analizzati i campioni fecali, per un esame coproparassitologico standard (ECPS, corrispondente all'O\&P [ova and parasites] degli Autori anglosassoni), di 661 soggetti, di cui 440 adulti (188 maschi e 252 femmine) e 221 bambini di età compresa tra 1 e 16 anni (121 maschi e 100 femmine). Per ciascun soggetto pediatrico sono stati analizzati da 1 a 6 campioni fecali raccolti a giorni alternati (con una media di 3.3 campioni per soggetto); per ciascun soggetto adulto sono stati analizzati da 1 a 6 campioni (con una media di 2.6 campioni per soggetto).

L'ECPS consisteva nella valutazione macroscopica dei singoli campioni fecali, nelle osservazioni microscopiche dirette (sia in soluzione fisiologica che in soluzione iodata) e dopo arricchimento formolo-etileacetato (FEA) dei medesimi, e osservazioni microscopiche dopo colorazioni permanenti (colorazione di Giemsa usualmente, ma pure colorazione tricromica e colorazione Ziehl Neelsen all'occorrenza), laddove indicato dalle valutazioni preliminari sia clinico-anamnestico-epidemiologiche sia, soprattutto, microscopiche (4). L'ECPS era mirato alla individuazione di tutti i protozoi patogeni e non patogeni di pertinenza umana e alla ricerca delle uova (o larve) di tutti gli elminti, esclusione fatta per Enterobius vermicularis e Strongyloides stercoralis $(4,16)$.

Per la ricerca mirata di E. vermicularis si utilizzò la tecnica di Graham (o dello scotch-test), che fu condotta su 40 soggetti pediatrici e su 6 soggetti adulti $(4,20)$.

La ricerca specifica di $S$. stercoralis fu condotta, utilizzando il metodo di Baermann modificato e/o la coltura delle larve su agar, su 42 soggetti adulti, sulla base anche di razionali indicazioni al riguardo $(18,22)$.

Le motivazioni alle indagini coproparassitologiche specifiche verranno dettagliatamente descritte successivamente nel corso della presentazione dei risultati e della loro discussione.

\section{RISULTATI E DISCUSSIONE}

I risultati complessivi sono riportati in tabella 1. Le positività sono state di 91 soggetti parassitati, pari al $13.8 \%$ dei totali analizzati in tale studio; in particolare 30 nei bambini (13.5\%) e 61 negli adulti $(13.9 \%)$, con leggere prevalenze nei maschi rispetto alle femmine (tabella 1 ). In tabella 2 vengono presentate le positività (in bambini ed in adulti, in maschi ed in femmine) per i parassiti patogeni (uno o più per soggetto, in caso con presenza anche di parassiti non ritenuti patogeni) e per i parassiti cosiddetti non patogeni (uno o più per soggetto). Così, tra i bambini, il 7.2\% è risultato parassitato da protozoi e/o elminti patogeni (con leggerissima maggior frequenza nei maschi) e il $6.3 \%$ da non patogeni; tra gli adulti, il $9.6 \%$ e il $4.3 \%$ rispettivamente, con maggior frequenza nei maschi per i patogeni e modesta maggior frequenza nelle femmine per $i$ non patogeni. Complessivamente, $1^{\prime} 8.8 \%$ dei soggetti era parassitato da forme patogene e il $5.0 \%$ da forme non patogene, con lieve prevalenza nei maschi per le prime e nelle femmine per le seconde (tabella 2). In tabella 3 vengono riportate le eziologie delle parassitosi sostenute da forme patogene.

Hanno prevalso le protozoosi rispetto alle elmintiasi, sia nel gruppo dei bambini (4.6\% versus $2.7 \%)$ sia nel gruppo degli adulti $(7.5 \%$ versus $2.3 \%$ ), per un totale complessivo del $6.5 \%$ versus il $2.4 \%$ (tabella 3 ). In particolare ha prevalso Dientamoeba fragilis, con 24 casi, pari al 3.6\%, seguita da Giardia duodenalis con 17 casi (2.6\%), sebbene quest'ultima abbia prevalso sulla prima nei soggetti pediatrici $(1.8 \%$ versus $0.5 \%)$, mentre netta è stata la maggiore frequenza della dientamoebiasi nei confronti della giardiasi nella popolazione adulta (5.2\% versus $1.8 \%)$. Cryptosporidium spp. (C. hominis/parvum) è stato osservato soltanto in 2 soggetti, giovani adulti, "HIV positivi" (9). Per quanto riguarda gli elminti, nei bambini sono stati reperiti esclusivamente E. vermicularis $(0.9$ $\%$ ) e Trichuris trichiura (1.8\%), come mostrato in tabella 4. Negli adulti il ventaglio delle eziologie è stato più ampio con il reperimento di E. vermicularis $(0.5 \%)$, T. trichiura $(0.7 \%)$, S. stercoralis $(0.7 \%)$, Hymenolepis nana $(0.2 \%)$, Taenia saginata $(0.2 \%)$, e Schistosoma mansoni $(0.2 \%)$.

Debbono essere fatte alcune precisazioni al riguardo. Il reperimento di E. vermicularis riferito in tabella 4 (sempre in soggetti autoctoni) è stato casuale, vuoi di uova non embrionate nel FEA vuoi di vermi adulti frammisti al materiale fecale (20). Nella ricerca mirata di E. vermicularis, infatti, questo nematode è stato riscontrato in 9 bambini su 40 analizzati specificatamente (sem- 
pre tutti autoctoni), pari al 22.5\% (4 su 16 maschi, pari al $25.0 \%$, e 5 su 24 femmine, pari al $21.0 \%$ ); nella fattispecie $E$. vermicularis è stato riscontrato in 6 su 20 bambini con prurito anale $(30.0 \%) \mathrm{e}$ in 3 su 20 con altri disturbi, pari al 15.0\% (disturbi intestinali aspecifici e/o dolori addominali, prurito sistemico, orticaria, o asintomatici, solitamente controlli in contatti). Nell'unico soggetto adulto su $6(16.7 \%)$ in cui furono rinvenute uova di questo ossiuro, va detto che questo fu osservato nello scotch test dopo suo iniziale e casuale reperimento in un campione urinario.

Per quanto concerne invece $S$. stercoralis, le larve rabditoidi di quest'altro nematode furono osservate sia dopo FEA sia con la ricerca mirata. Quest'ultima fu condotta peraltro su 42 soggetti soprattutto anziani (sempre qualora autoctoni) in altrettante circostanze e le positività furono pertanto del 7.1\% (tutte in soggetti maschili). In due casi trattavasi di soggetti italiani (ma uno residente da sempre in Calabria ed un altro con pregresse residenze in svariati PVS in quanto missionario), nel terzo caso di soggetto (giovane adulto) proveniente dalla Costa d'Avorio.

I 4 casi pediatrici di trichuriasi furono osservati tutti in bambini non italiani, mentre i 3 casi adulti si riferivano a 2 soggetti italiani e a 1 soggetto proveniente dal Camerun. (tabella 5).

$H$. nana fu osservata in un soggetto egiziano, mentre i 2 casi di T. saginata furono osservati in soggetti italiani. La diagnosi di teniasi da $T$. saginata fu ottenuta con l'osservazione delle proglottidi emesse con le feci in associazione alle tipiche uova di Taenia spp. (4).

I due casi di schistosomiasi da $S$. mansoni furono osservati in un paziente egiziano (parassitato anche da $H$. nana e da altri parassiti non patogeni) e in un paziente proveniente proveniente dal Camerun (lo stesso parassitato pure da T. trichiu$r a$ [vedi anche tabella 5])

Furono reperiti anche alcuni casi di parassitosi spurie da Dicrocoelium dendriticum (uova di transito passivo, mai reperiti in successivi controlli): 2 in bambini (un italiano ed uno proveniente dall'Europa dell'Est) e 5 in adulti (di cui 4 in soggetti italiani autoctoni) (21).

Per quanto riguarda i protozoi patogeni, va precisato che 7 su 9 casi (77.8\%) di giardiasi osservati nei bambini erano di importazione (essenzialmente in controlli su bambini adottati; questi furono 20 e in 8 casi, pari al $40.0 \%$, furono repertate positività per parassiti), così come l'unico caso di dientamoebiasi, come riportato in tabella 5 (26). Per contro, negli adulti, tutti gli 8 casi di giardiasi, 19 casi su $23(83.0 \%)$ di dientamoebiasi e tutti i 2 casi di cryptosporidiasi erano autoctoni (17). Inoltre, potrebbe essere utile precisare che tra le parassitosi sostenute da forme non patogene, mentre erano prevalenti quelle di importazione (19 casi su 21, pari all'89\%) nei bambini, tra gli adulti parassiti non patogeni hanno prevalso nei soggetti autoctoni ( 29 su 40, pari al 73\%). Nel complesso, se nei bambini la maggior parte delle parassitosi è stata osservata in soggetti non italiani provenienti da aree endemiche di PVS (23 su 30, pari al 77\%), negli adulti si è osservato il contrario, ovvero la maggior parte dei parassiti era appannaggio di soggetti italiani e contratte verosimilmente nel nostro Paese ed in particolare nella nostra area geografica (51 su 61, pari all' $84 \%$ ). Nel complesso, così, il $64 \%$ delle parassitosi è stato ritenuto autoctono ed il $36 \%$ di importazione.

Debbono poi venire precisate le non infrequenti associazioni, osservate soprattutto tra parassiti patogeni e non patogeni ed all'interno dei parassiti non patogeni (essenzialmente protozoi). Nel gruppo dei bambini le associazioni tra 2 o più parassiti sono state osservate in 7 casi su 30 (23.3\%); nel gruppo degli adulti tali associazioni sono state osservate in 23 su 61 soggetti (37.7\%). Per quanto concerne i protozoi non patogeni, rilevati nel $5 \%$ dei soggetti, nella maggior parte dei casi trattatasi di Blastocystis hominis (sia casi autoctoni che verosimilmente importati) e più raramente di altre amebe non patogene, quali Endolimax nana, Entanoeba coli ed Entamoeba hartmanni (soprattutto di importazione); eccezionali sono stati i casi di flagellati commensali. In nessuna circostanza tali protozoi furono associati a quadri nosologici evidenti in quanto, se rilevati o segnalati, sono stati correlati alla simultanea presenza di parassiti francamente patogeni. Non è stato inoltre ritenuto razionale, in quanto aleatorio e soggettivo, valutare l'entità della presenza di qualsivoglia protozoo saprofita (o in ogni caso non ritenuto patogeno) in termini di quantizzazione numerica microscopica.

Le motivazioni alle indagini coproparassitologiche (ECPS) sono state le più varie (purtroppo non sempre specificate nel dettaglio), quali disturbi intestinali aspecifici (nei bambini più spesso dolori addominali ricorrenti), ipereosinofilia periferica, prurito cutaneo diffuso, lesioni o patologie cutanee varie (sovente orticaria), intolleranza alimentare, controlli per motivi svariati (in soggetti asintomatici o apparentemente tali e talora in bambini con ritardo di sviluppo di crescita). Ebbene il 14.1\% dei soggetti pediatrici con dolori intestinali aspecifici ed il $10.3 \%$ degli adulti con disturbi intestinali aspecifici era parassitato da patogeni o non patogeni; il $21.7 \%$ dei soggetti adulti con ipereosinofilia è risultato positivo per una parassitosi, e spicca in particolare il $10.9 \%$ di positività per $D$. fragilis; il $20.0 \%$ dei bambini e il $20.8 \%$ degli adulti con prurito cutaneo erano 
altresì positvi per una parassitosi, mentre lo furono nel $16.0 \%$ e $7.8 \%$ rispettivamente nel gruppo con patologie cutanee; infine parassiti furono individuati nel $24.3 \%$ dei bambini e nel $15.5 \%$ degli adulti asintomatici o apparentemente tali per i quali l'ECPS fu richiesto per controllo, spesso non meglio precisato (vedi tabelle 6 e 7).

Vogliamo ribadire l'importanza di approcci metodologici diagnostici differenziati per l'ECPS, per la ricerca mirata di $S$. stercoralis, e, soprattutto, per la ricerca finalizzata di E. vermicularis $(4,20,22)$. Ed è quanto mai doveroso, all'interno dell'ECPS, eseguire sempre o potere eseguire quando necessaria la colorazione di Giemsa per l'identificazione di $D$. fragilis ed una colorazione all'acido-resistenza (Ziehl-Neelsen o analoga) per Cryptosporidium spp., utile, quest'ultima, anche per gli altri coccidi usualmente importati, in particolare Cyclospora cayetansensis $(5,17,27)$.

Sicuramente, mentre le parassitosi, in particolar modo le protozoosi, nei soggetti pediatrici sono soprattutto di importazione (bambini adottati in primis), le medesime, negli adulti, sono prevalentemente autoctone (tabella 5). L'iter diagnostico, e non soltanto strettamente diagnostico, deve essere peraltro del tutto sovrapponibile, per cui ci è parso corretto presentare $\mathrm{i}$ risultati nel loro insieme e soltanto successivamente esplicare le differenze intrinseche osservate in tali gruppi di popolazioni e in relazione ai vari quadri nosologici riferiti $(6,15,19)$.

Tra gli elminti, se E. vermicularis in particolar modo, e $S$. stercoralis, con frequenze minori, sono tuttora presenti nella nostra area geografica (o comunque in Italia), gli altri nematodi, cestodi e trematodi sono indubbiamente rari, seppure non scomparsi o forse riapparsi $(15,18,20)$. Lo testimoniano soprattutto i due casi di teniasi e quei pochi casi di tricocefalosi osservati, anche se in tale casistica, ad esempio, non figuri neppur un caso di ascaridiasi (10). Il reperimento di uova di elminti da noi mai presenti (S. mansoni), o di elminti ritenuti da sempre assenti (nell'anno precedente furono trovate uova di $O$. felineus in una copia di giovani adulti [15]; reperto confermato in altro soggetto ai primi del 2006, tuttora in corso di pubblicazione), nonché di uova di transito di $D$. dendriticum (in quanto come tali ingerite passivamente in seguito a consumo solitamente di "coratella d'agnello"), sono tutti elementi che debbono garantire attenzione e capacità diagnostiche non così scontate. Alcune uova, come quelle di O. felineus, ad esempio, sono molto piccole e possono sfuggire all'esame microscopico; altre uova, ancora piccole ma di più semplice individuazione, e non facili sempre da identificare nella specie, debbono sempre essere ricontrollate per escludere probabili false parassitosi, come nei casi riferiti di dicrocaeliasi umana spuria (21). In tale casistica di un anno, i tre casi di $S$. stercoralis sono stati del tutto fortuitamente osservati anche dopo FEA. Ma questo deve fare riflettere sul fatto che molti campioni fecali di soggetti "a rischio" per una potenziale strongyloidiasi erano quantitativamente insufficienti per una ricerca mirata delle larve di tale nematode; e purtroppo, nonostante questo fosse stato segnalato nel referto, mai o quasi mai da parte del curante è stato inviato almeno un ulteriore campione per verificare tale patologia parassitaria, che, in Umbria, è presente e forse tuttora sottostimata (18). Ancora, il reperimento di larve nei campioni fecali dopo FEA non sempre è espressione di una strongyloidiasi. Debbono riconoscersi le caratteristiche delle larve e il loro stadio e si deve essere in grado di non confonderle con larve di nematodi a vita libera passivamente introdotti con gli alimenti (e a noi almeno un caso è occorso e riferivasi ad un soggetto che, avendo consumato tartufi, verosimilmente aveva ingerito anche larve di nematodi tutt'altro che di rado contaminanti questi tuberi) (24). Ecco quindi l'importanza di procedere ulteriormente, con metodi idonei (Baermann, analoghi o complementari), per una diagnosi differenziale quanto mai delicata $(4,22)$.

Per quanto riguarda $E$. vermicularis e scotch test, si ribadisce la necessità di analizzare sempre almeno tre campioni (4). Questo è da correlare non tanto alla biologia dell'ossiuro, quanto alla scarsa agevolezza nel raccogliere il campione, con inevitabili rischio di false negatività (20). Nell'esperienza qui riportata, a mò di esempio, per quanto concerne i 9 campioni di scotch test positivi per uova di $E$. vermicularis, soltanto in 5 casi la presenza di queste uova fu osservata al I campione, mentre in 3 casi la positività apparve al II ed i 1 caso soltanto al III vetrino analizzato (e i tre campioni erano tutti positivi solamente in 2 circostanze) (20).

In tema di protozoosi, se è pur vero che Cryptosporidium spp. è assai raro e che G. duodenalis nei bambini è soprattutto importata, è altresì vero che la stessa circola in Italia, e non è così rara, sebbene, in ogni caso, sia meno frequente di $D$. fragilis, tutt'altro che infrequente, e della stessa Blastocystis hominis, usualmente ritenuto protozoo non patogeno (o saprofita opportunista), che spesso è associato proprio agli ultimi due e/o a protozoi sicuramente saprofiti, espressione probabilmente di contaminazioni idroalimentari o ambientali $(4,5)$. B. hominis è stata osservata essenzialmente negli adulti con una frequenza complessiva del 5.9\% (in modo pressoché eguale in maschi e femmine), seppure da sola sia stata reperita solamente nel $2.0 \%$ dei soggetti (in assenza di sintomatologia evidente o come tale 
riferita). Colpisce in definitiva, tra tutti questi, la più elevata frequenza di $D$. fragilis, sicuramente patogeno e potenziale tale, osservabile tanto nei maschi quanto nelle femmine, anche se soprattutto negli adulti, talora associato ad altri parassiti, e responsabile di quadri clinici aspecifici o variabili (non di rado un'ipereosinofilia), fermo restando che è anche spesso in causa in diarree acute o protratte, ma che in tale casistica non sono state analizzate $(11,17)$. Da esperienze personali "sul campo" (in altri termini presso altre strutture diagnostiche presso le quali abbiamo potuto operare o con le quali ci siamo confrontati), è emerso come sovente $D$. fragilis (e la stessa $B$. hominis) vengano confuse, a causa della mancata applica- zione di colorazioni idonee, con "amebe non patogene", in particolare E. coli e non meglio specificate Entamoeba spp.

Per concludere in modo estremamente sintetico, ma efficace, anche in virtù di quanto sinora esposto, vogliamo sottolineare che le parassitosi intestinali, sia quelle sostenute da protozoi sia quelle sostenute da elminti, pur non frequenti come altre patologie microbiche, sono ancora presenti, in parte autoctone, in parte importate. E' così necessaria una impostazione diagnostica metodologica ragionata, razionale e mirata, nonché attenta, responsabile e competente, al fine di evitare pericolose sottostime e false negatività, troppo facilmente e a volte inconsciamente repertate e refertate.

Tabella I. Risultati complessivi all'Esame coproparassitologico standard (ECPS)

\begin{tabular}{|l|ll|lr|rr|}
\hline \multicolumn{1}{|c|}{ Soggetti parassitati } & \multicolumn{2}{|c|}{ MASCHI } & \multicolumn{2}{|c|}{ FEMMINE } & \multicolumn{2}{c|}{ TOTALI } \\
\hline Bambini & $17 / 121$ & $14.0 \%$ & $13 / 100$ & $13.0 \%$ & $30 / 221$ & $13.5 \%$ \\
\hline Adulti & $31 / 188$ & $16.5 \%$ & $30 / 252$ & $11.9 \%$ & $61 / 440$ & $13.9 \%$ \\
\hline Complessivi & $48 / 309$ & $15.5 \%$ & $43 / 352$ & $12.2 \%$ & $91 / 661$ & $13.8 \%$ \\
\hline
\end{tabular}

Tabella 2. Prospetto dei risultati discriminando tra positività per parassiti patogeni e non patogeni (all'ECPS)

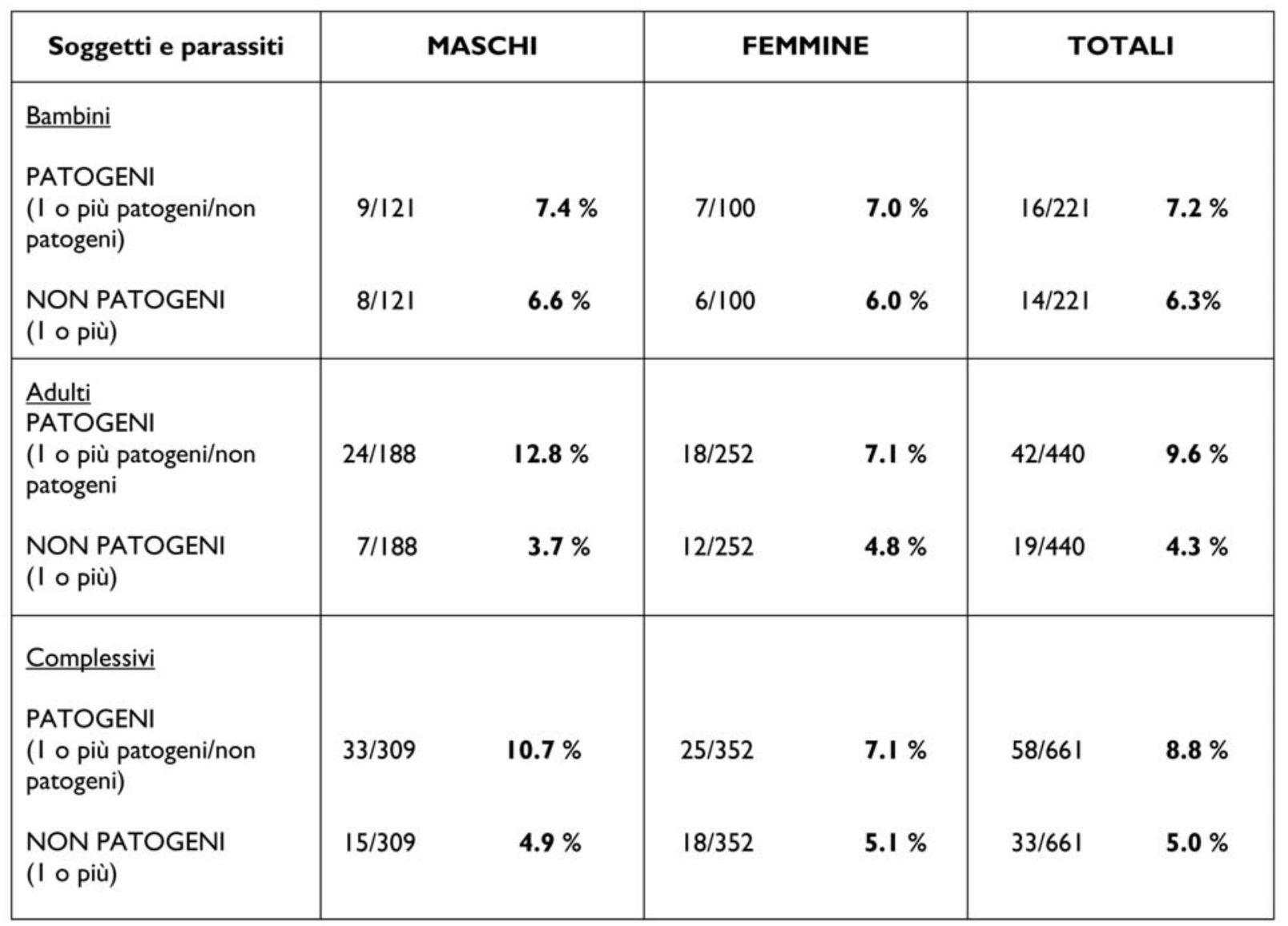


Tabella 3. Eziologie parassitarie e soggetti colpiti (ECPS)

\begin{tabular}{|c|c|c|c|c|c|c|}
\hline $\begin{array}{l}\text { PARASSITI } \\
\text { PATOGENI }\end{array}$ & \multicolumn{2}{|c|}{ MASCHI } & \multicolumn{2}{|c|}{ FEMMINE } & \multicolumn{2}{|c|}{ TOTALI } \\
\hline G. duodenalis & $4 / 121$ & $3.3 \%$ & $5 / 100$ & $5.0 \%$ & $9 / 221$ & $4.1 \%$ \\
\hline D. fragilis & $1 / 121$ & $0.8 \%$ & $0 / 100$ & - & $1 / 221$ & $0.5 \%$ \\
\hline ELMINTI & $4 / 121$ & $3.3 \%$ & $2 / 100$ & $2.0 \%$ & $6 / 221$ & $2.7 \%$ \\
\hline \multicolumn{7}{|l|}{ Adulti } \\
\hline G. duodenalis & $5 / 188$ & $2.7 \%$ & $3 / 252$ & $1.2 \%$ & $8 / 440$ & $1.8 \%$ \\
\hline D. fragilis & $11 / 188$ & $5.9 \%$ & $12 / 252$ & $4.8 \%$ & $23 / 440$ & $5.2 \%$ \\
\hline C. hominis/parvum & $1 / 188$ & $0.5 \%$ & $1 / 252$ & $0.4 \%$ & $2 / 440$ & $0.5 \%$ \\
\hline ELMINTI * & $8 / 188$ & $4.3 \%$ & $2 / 252$ & $0.8 \%$ & $10 / 440$ & $2.3 \%$ \\
\hline \multicolumn{7}{|l|}{ Complessivi } \\
\hline G. duodenalis & $9 / 309$ & $2.9 \%$ & $8 / 352$ & $2.3 \%$ & $17 / 661$ & $2.6 \%$ \\
\hline D. fragilis & $12 / 309$ & $3.9 \%$ & $12 / 352$ & $3.4 \%$ & $24 / 661$ & $3.6 \%$ \\
\hline C. hominis/parvum & $1 / 309$ & $0.3 \%$ & $1 / 352$ & $0.3 \%$ & $2 / 661$ & $0.3 \%$ \\
\hline ELMINTI & $12 / 309$ & $3.9 \%$ & $1 / 352$ & $0.3 \%$ & $16 / 661$ & $2.4 \%$ \\
\hline
\end{tabular}

Note: * I caso associato a $D$. fragilis

Tabella 4. Elminti patogeni reperiti nella popolazione esaminata (all'ECPS)

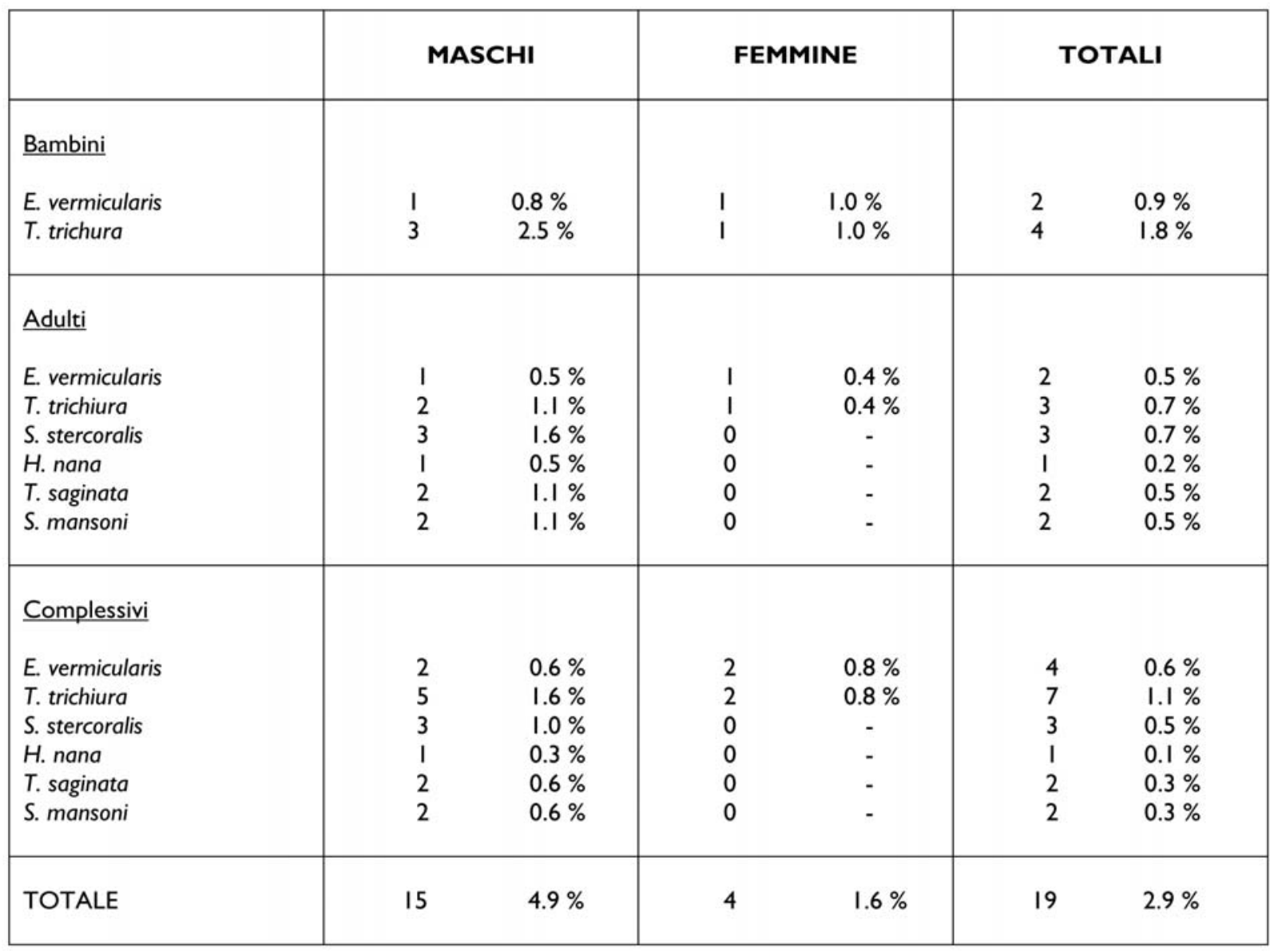


Tabella 5. Descrizione delle frequenze delle parassitosi autoctone ed importate in bambini ed adulti

\begin{tabular}{|c|c|c|c|c|c|c|c|c|}
\hline \multirow{3}{*}{$\begin{array}{l}\text { PARASSITA / PARASSITOSI }{ }^{\circledR} \\
\text { TM } \\
\text { G. duodenalis }\end{array}$} & \multicolumn{4}{|c|}{ AUTOCTONE } & \multicolumn{4}{|c|}{ DI IMPORTAZIONE } \\
\hline & \multicolumn{2}{|c|}{ Bambini } & \multicolumn{2}{|c|}{ Adulti } & \multicolumn{2}{|c|}{ Bambini } & \multicolumn{2}{|c|}{ Adulti } \\
\hline & $2 / 9$ & $22.2 \%$ & $8 / 8$ & $100 \%$ & $7 / 9$ & $77.8 \%$ & $0 / 8$ & - \\
\hline D. fragilis & $0 / 1$ & - & $19 / 23$ & $82.6 \%$ & I / I & $100 \%$ & $4 / 23$ & $17.4 \%$ \\
\hline Protozoi/elminti non patogeni & $2 / 21$ & $9.5 \%$ & $29 / 40$ & $72.5 \%$ & $19 / 21$ & $90.5 \%$ & $11 / 40$ & $27.5 \%$ \\
\hline Elminti patogeni & $3 / 8$ & $37.5 \%$ & $13 / 18$ & $72.2 \%$ & $5 / 8$ & $62.5 \%$ & $5 / 18$ & $27.8 \%$ \\
\hline Complessivi & $7 / 39$ & $17.9 \%$ & $69 / 89$ & $77.5 \%$ & $14 / 39$ & $35.9 \%$ & $20 / 89$ & $22.5 \%$ \\
\hline
\end{tabular}

Tabella 6. Correlazione tra parassitali e quadri nosologici nella popolazione pediatrica

\begin{tabular}{|c|c|c|c|c|c|c|c|c|c|}
\hline & \multicolumn{2}{|c|}{$\begin{array}{l}\text { DOLORI } \\
\text { ADDOMINALI } \\
\text { RICORRENTI/ } \\
\text { DISTURBI } \\
\text { INTESTINALI } \\
\text { ASPECIFICI }\end{array}$} & \multirow[t]{2}{*}{ IPERESOSINOFILIA } & \multicolumn{2}{|c|}{ PRURITO } & \multicolumn{2}{|c|}{$\begin{array}{l}\text { ORTICARIA, } \\
\text { ALLERGIA O } \\
\text { ALTRI } \\
\text { DISTURBI } \\
\text { CUTANEI }\end{array}$} & \multicolumn{2}{|c|}{$\begin{array}{l}\text { ALTRO } \\
\text { (CONTROLLI, } \\
\text { ASINTOMATICI, } \\
\text { NON RIFERITO) }\end{array}$} \\
\hline G. duodenalis & 1 & $0.9 \%$ & & & $20.0 \%$ & & & 7 & $10.0 \%$ \\
\hline D. fragilis & 1 & $0.9 \%$ & & & & & & & \\
\hline B. hominis & 2 & $1.8 \%$ & & & & & & & \\
\hline $\begin{array}{l}\text { Altri } \\
\text { protozoi } \\
\text { (non } \\
\text { patogeni) }\end{array}$ & 5 & $4.4 \%$ & & & & 3 & $12.0 \%$ & 10 & $14.3 \%$ \\
\hline Elminti & 7 & $6.1 \%$ & & & & 1 & $4.0 \%$ & & \\
\hline TOTALI & $16 / 114$ & $14.1 \%$ & $0 / 7$ & $1 / 5$ & $20.0 \%$ & $4 / 25$ & $16.0 \%$ & $17 / 70$ & $24.3 \%$ \\
\hline
\end{tabular}


Tabella 7. Correlazione tra parassiti e quadri nosologici nella popolazione adulta

\begin{tabular}{|c|c|c|c|c|c|c|c|c|c|c|}
\hline \multirow[b]{2}{*}{ G. duodenalis } & \multicolumn{2}{|c|}{$\begin{array}{l}\text { DISTURBI } \\
\text { INTESTINALI } \\
\text { ASPECIFICI }\end{array}$} & \multicolumn{2}{|c|}{$\begin{array}{l}\text { IPEREOSINOFILIA } \\
\text { (CON O SENZA } \\
\text { PRURITO / ET } \\
\text { AL.) }\end{array}$} & \multicolumn{2}{|c|}{$\begin{array}{l}\text { PRURITO } \\
\text { DIFFUSO } \\
\text { (CON O } \\
\text { SENZA } \\
\text { LESIONI } \\
\text { CUTANEE) }\end{array}$} & \multicolumn{2}{|c|}{$\begin{array}{l}\text { LESIONI } \\
\text { CUTANEE } \\
\text { (ORTICARIA } \\
\text { ET AL.) }\end{array}$} & \multicolumn{2}{|c|}{$\begin{array}{l}\text { ALTRO } \\
\text { (CONTROLLI } \\
\text { ASINTOMATICI } \\
\text { NON } \\
\text { RIFERITO) }\end{array}$} \\
\hline & $6^{\circ}$ & $2.7 \%$ & & & & & & & 2 & $3.4 \%$ \\
\hline D. fragilis & 8 & $3.6 \%$ & $5 *$ & $10.9 \%$ & 4 & $8.3 \%$ & 2 & $3.1 \%$ & 4 & $6.9 \%$ \\
\hline B. hominis & 3 & $1.3 \%$ & & & 4 & $8.3 \%$ & 1 & $1.6 \%$ & 1 & $1.8 \%$ \\
\hline $\begin{array}{l}\text { Altri } \\
\text { protozoi } \\
\text { (non } \\
\text { patogeni) }\end{array}$ & 4 & $1.8 \%$ & 1 & $2.2 \%$ & & & 2 & $3.1 \%$ & & \\
\hline Elminti & 2 & $0.9 \%$ & 4 & $8.7 \%$ & 2 & $4.2 \%$ & & & 2 & $3.4 \%$ \\
\hline TOTALI & $23 / 224$ & $10.3 \%$ & $10 / 46$ & $21.7 \%$ & $10 / 48$ & $20.8 \%$ & $5 / 64$ & $7.8 \%$ & 9/58 & $15.5 \%$ \\
\hline
\end{tabular}

\section{BIBLIOGRAFIA}

1. Barbaro GB, Cavallino AM, Gianotti A, Patrone C, Robello A. Esami coproparassitologici: analisi di una casistica quinquennale raccolta nel territorio del Ponente Genovese. GIMMOC 2001; Vol. V N 1: 1820.

2. Bernieri F, Galli D, Viti F, et al. Parassitosi enteriche autoctone. Microbiol. Med. 1994; 9: 415-418.

3. Bernieri F, Galli D, Giordano S, et al. Indagine nazionale AMCLI-CoSP sulla diffusione delle parassitosi intestinali. Microbiol. Med. 1996; 11: 463-472.

4. Bernieri F, Crotti D, Galli D, Raglio A. Manuale illustrato di diagnostica parassitologica. Selecta Medica, Pavia, 2001.

5. Bernieri F, Crotti D. Infezioni da protozoi. Microbiol. Med. 2001; 16: 98-108.

6. Bernieri F, Casella P, Crotti D, et al. Linee Guida Operative per la diagnosi delle parassitosi intestinali. Microbiol. Med. 2005; 20: 39-46.

7. Bethony J, Brooker S, Albonico M, et al. Soil-transmitted helminth infections: ascariasis, trichuriasis, and hookworm. Lancet 2006; 367: 1521-1532.

8. Caruana S R, Kelly H A, Ngeow J Y Y, et al. Undiagnosed and potentially lethal parasite infections among immigrants and refugees in Australia. J. Travel Med. 2006; 13: 233-239.

9. Crotti D. Protozoosi nel paziente immuno-compromesso. De Re Medica 1993; 2: 7-8.

10. Crotti D, De Angelis L, Del Sante M, Fonzo G, De Giorgi G. Parassitosi intestinali: incidenze annuali e descrizione di un raro caso di imenolepiasi.
Microbiol. Med. 1996; 11: 381-382.

11. Crotti D, D’Annibale ML. Dientamoeba fragilis e dientamoebosi: aspetti di parassitologia clinica e diagnostica di laboratorio. Parassitologia 2001; 43: 135138.

12. Crotti D. Parassitosi intestinali autoctone nella seconda metà degli anni '90: considerazioni critiche diagnostiche. Microbiol. Med. 2002; 17: 7-13.

13. Crotti D, D’Annibale ML, Medori MC, Fonzo G, Ubaldi M. Parassitosi intestinali autoctone nell'area del Perugino: considerazioni di metodologia diagnostica e prevalenze nel corso del 2001. GIMMOC 2003; Vol. VII N 1: 17-20.

14. Crotti D, Raglio A, Galli D, Bernieri F. L'esame coproparassitologico nei bambini. Indicazioni e indagini raccomandate. Quaderni acp 2004; 12: 39-42.

15. Crotti D, Fioravanti M L, Gustinelli A, Florio D, Pampiglione S. Two cases of human Opistorchiasis in Italy. Parassitologia 2004; 46: 111.

16. Crotti D, D’Annibale M.L. Strongyloidiasi nel triennio 2001-2003: considerazioni diagnostiche e clinicoepidemiologiche. GIMMOC 2004; Vol. VIII No 1 : 1523.

17. Crotti D, D’Annibale M L, Fonzo G, Lalle M. Cacciò $\mathrm{S}$ M, Pozio E. Dientamoeba fragilis is more prevalent than Giardia duodenalis in children and adults attending a day care centre in Central Italy. Parasite 2005; 12: $165-170$.

18. Crotti D, D'Annibale ML, Rossi S. Strongyloides stercoralis e strongiloidiasi in Umbria: analisi di una casistica decennale. Microbiol. Med. 2005; 20: 250256. 
19. Crotti D, Bernieri F, Raglio A, Galli D. L'esame coproparassitologico: indicazioni di base per una adeguata diagnostica. Rivista SIMG 2005; 5-5: 28-32.

20. Crotti D, D'Annibale ML. Enterobiasi nel biennio 2002-2003 nel perugino: considerazioni non soltanto diagnostiche. Le Infezioni in Medicina 2006; 2: $92-$ 98.

21. D’Annibale M L. Infestazione spuria da Dicrocoelium dendriticum. Microbiol. Med. 2004; 19: 399-400.

22. Del Sante M, Fonzo G, Crotti D. Infestazioni autoctone da Strongyloides stercoralis: quale diagnostica e quale endemia. Microbiol. Med. 1997; 12: 456-458.

23. Giordano S, Troia G, Miraglia P, Scarlata F. Epidemiologia delle parassitosi intestinali in Sicilia occidentale. Osservazioni relative agli anni 19932000. Le Infezioni in Medicina 2001; 3: 154-157.

24. Granetti B, De Angelis A, Materozzi G. Umbria terra di tartufi. Regione Umbra Edizioni, Perugia, 2005; 40.

25. Lainson R, Da Silva B A M. Intestinal parasites of some diarrhoeic HIV-seropositive individuals in North Brasil, with particolar reference to Isospora belli Wenyon, 1923 and Dientamoeba fragilis Jepps \& Dobell, 1918. Mem. Inst. Oswaldo Cruz, 1999; 94: 611-613.

26. Ricci E, De Michele M B. Valutazione dell'incidenza di parassitosi sostenute da Giardia intestinalis in una popolazione compresa tra la Valle Peligna e l'alta Val Pescara dopo l'emigrazione di diversi nuclei familiari (circa 200 persone) provenienti dall'Albania avvenuta negli anni 90 (1997-1999). Riv. Med. Lab. 2001; 2: 15-16.

27. Scaglia M, Gatti S, Viale P, Bassi P, Novati S, Ranieri S. Enterite diarroica da Cyclospora spp., un nuovo coccidio patogeno per l'uomo: descrizione di un caso in paziente con AIDS e revisione della letteratura. Microbiol. Med. 1994; 9: 9-11.

28. Utzinger J, N'Goran EK, Marti HP, Tanner M, Lengeler C. Intestinal amoebiasis, giardiasis and geohelminthiases: their association with other intestinal parasites and reported intestinal symptoms. Trans. R. Soc. Trop. Med. Hyg. 1999; 93: 137-141.

\author{
Daniele Crotti \\ Strada Comunale per Pilonico Paterno 4, \\ 06080 Pianello, Perugia \\ Tel.: +39075 602372 \\ E-mail: nenedc@tin.it
}

